\title{
Review \\ Medications for analgesia and sedation in the intensive care unit: an overview
}

\author{
Diederik Gommers and Jan Bakker
}

Department of Intensive Care, Erasmus MC, Gravendijkwal 230, 3015 CE Rotterdam, The Netherlands

Corresponding author: Diederik Gommers, d.gommers@erasmusmc.nl

Published: 14 May 2008

This article is online at http://ccforum.com/content/12/S3/S4

(c) 2008 BioMed Central Ltd
Critical Care 2008, 12(Suppl 3):S4 (doi:10.1186/cc6150)

cutaneous and parenteral routes. However, delayed gastric emptying, (continuous) gastric drainage, decreased gut function, uncertain first-pass effect, general edema, and use of vasopressors, usually make the parenteral route preferable in critically ill patients. Parenteral drugs can be given by continuous infusion, bolus and patient-controlled analgesic techniques. In addition, epidural infusions of local anesthetics, often combined with opioids, can provide effective analgesia, for example in the case of fractured ribs or thoracic or abdominal wounds.

\section{Epidural analgesia}

Epidural analgesia is used extensively in modern anesthetic practice. A meta-analysis of more than 5,000 surgical patients [4] has shown that postoperative epidural analgesia reduces time to extubation, ICU stay, incidence of renal failure, morphine consumption during the first 24 hours, and maximal glucose and cortisol blood concentrations, and improves forced vital capacity. Many of these benefits may also be relevant to ICU patients, as has been demonstrated for cardiac surgical [5] and thoracic trauma [6] patients. The effectiveness and safety of epidural analgesia has also been demonstrated in critically ill patients with severe acute pancreatitis [7]. However, whether sepsis, with or without positive blood cultures, is an absolute contraindication for the use of epidural analgesia is still a matter of debate. Further research is therefore required to define the role of epidural analgesia in this high-risk group [8].

\section{Opioids}

Opioids remain the mainstay drug for analgesic therapy in the ICU patient [9]. Opioids act selectively on neurons that transmit and modulate nociception, leaving other sensory modalities and motor functions intact. Opioid receptors are found in the brain, spinal cord, and peripheral tissues. All opioids depress respiratory drive in a dose-dependent manner, and this depression is increased when opioids are given in

$\mathrm{CNS}=$ central nervous system; $\mathrm{GABA}=\gamma$-aminobutyric acid $; \mathrm{ICU}=$ intensive care $u$ unit. 
combination with benzodiazepines. In general, opioids have minimal hemodynamic effects in euvolemic patients. Dependence and withdrawal effects can occur with prolonged continuous infusion of opioids.

\section{Morphine}

Morphine is the oldest opioid in current use, being discovered almost 200 years ago, and it is the most hydrophilic of the opioids. The dose required to produce analgesia varies markedly and depends on factors such as tolerance, and metabolic and excretory ability. Hepatic metabolism produces 80\% morphine-3-glucuronide (which has no analgesic action or neurotoxicity) and $20 \%$ morphine-6-glucuronide. The latter is a potent analgesic, having 20 to 40 times the activity of morphine $[10,11]$. Both metabolites are eliminated by the kidney and may therefore accumulate in patients with renal dysfunction. For this reason, fentanyl is preferred in patients with renal insufficiency [12]. Morphine-induced histamine release is rarely clinically important in ICU patients.

\section{Fentanyl and remifentanil}

Fentanyl and sufentanil are fat soluble and therefore have a rapid onset of action. However, increased fat solubility also results in increased volume of distribution, thereby increasing the risks for accumulation and delayed recovery after prolonged administration. In contrast, remifentanil (a derivative of fentanyl) is a short-acting opioid with unique properties. Remifentanil is metabolized by unspecific esterases that are widespread throughout the plasma, red blood cells, and interstitial tissues, whereas elimination of other opioids requires hepatic biotransformation and renal excretion. The context-sensitive half-time (the time required for the drug's plasma concentration to decrease by $50 \%$ after cessation of an infusion) of remifentanil is consistently short (3.2 minutes), even after an infusion of long duration ( $>8$ hours) [13]. Therefore, analgesia-based sedation with remifentanil has been introduced as an option in ICU patients.

Muellejans and colleagues [14] compared the efficacy and safety of remifentanil and fentanyl in postsurgical ICU patients. Both agents were effective in achieving the targeted sedation level and recovery was rapid, without any apparent difference between agents. In contrast, in their comparison of a remifentanil-based versus a morphine-based regimen under similar conditions, Dahaba and coworkers [15] found that the mean duration of mechanical ventilation and extubation time were significantly shorter in the remifentanil group. In critically ill patients requiring prolonged mechanical ventilation for up to 10 days, Breen and colleagues [16] compared an analgosedation regimen using remifentanil versus a midazolambased sedation regimen to which fentanyl or morphine was added for analgesia. The remifentanil-based sedation regimen was associated with significantly reduced duration of mechanical ventilation by more than 2 days. Although remifentanil is only licensed for up to 3 days of use in the ICU, it has been demonstrated even in such ICU patients (with an expected duration of mechanical ventilation of 2 to 3 days) that remifentanil-based analgosedation significantly decreases the duration of mechanical ventilation and length of ICU stay [17]. In addition, remifentanil does not exert significantly prolonged clinical effects when it is administered to ICU patients with renal failure $[18,19]$. Based on these studies, it can be concluded that remifentanil is effective for providing both analgesia and sedation in critically ill patients, even those suffering from multiple organ failure.

\section{Sedation}

A widely accepted theory regarding the action of anesthetics is that they interact with the inhibitory $\gamma$-aminobutyric acid (GABA) neurotransmitter system. The GABA and adrenergic neurotransmitter systems counterbalance the action of excitatory neurotransmitters. Hypnotic drugs interact with different components of the GABA receptor complex (Fig. 1).

\section{Benzodiazepines}

Benzodiazepines bind to a specific receptor site of the GABA receptor, and thus the degree of modulation is limited, which explains the 'ceiling effect' of their central nervous system (CNS) depression. It has been suggested that a benzodiazepine receptor occupancy of $20 \%$ provides anxiolysis, whereas an occupancy of $30 \%$ to $50 \%$ is associated with sedation, and $60 \%$ is required for hypnosis [20]. Benzodiazepines produce dose-dependent respiratory depression. In healthy patients, respiratory depression is moderate but depression is enhanced in patients with chronic respiratory disease, and synergistic depressant effects occur when benzodiazepines are combined with opioids.

Midazolam and lorazepam are the most commonly used benzodiazepines for ICU sedation [9]. Midazolam is a shortacting, water-soluble benzodiazepine that undergoes extensive oxidation in the liver via the cytochrome P450 enzyme system to form water-soluble hydroxylated metabolites, which are excreted in urine. However, the primary metabolite, namely 1 -hydroxymethylmidazolam, has mild CNS depressant activity and may accumulate in the critically ill patient, especially in the case of kidney failure. Medications that interfere with the cytochrome P450 enzyme (for example, erythromycin, calcium channel blockers, and itraconzole) will decrease metabolization of midazolam. Lorazepam is a longacting benzodiazepine that is metabolized by hepatic glucuronidation to inactive metabolites that are cleared by the kidneys. Hepatic dysfunction has greater impact on oxidation than glucuronidation.

During short-term infusions, both lorazepam and midazolam are generally safe and effective sedative agents. However, during continuous infusions accumulation of midazolam can occur because of the large volume distribution and its high lipophilicity. High-dose lorazepam treatment carries a risk for toxicity from propylene glycol, a vehicle that is used for solubility in the intravenous solution. It should be considered 


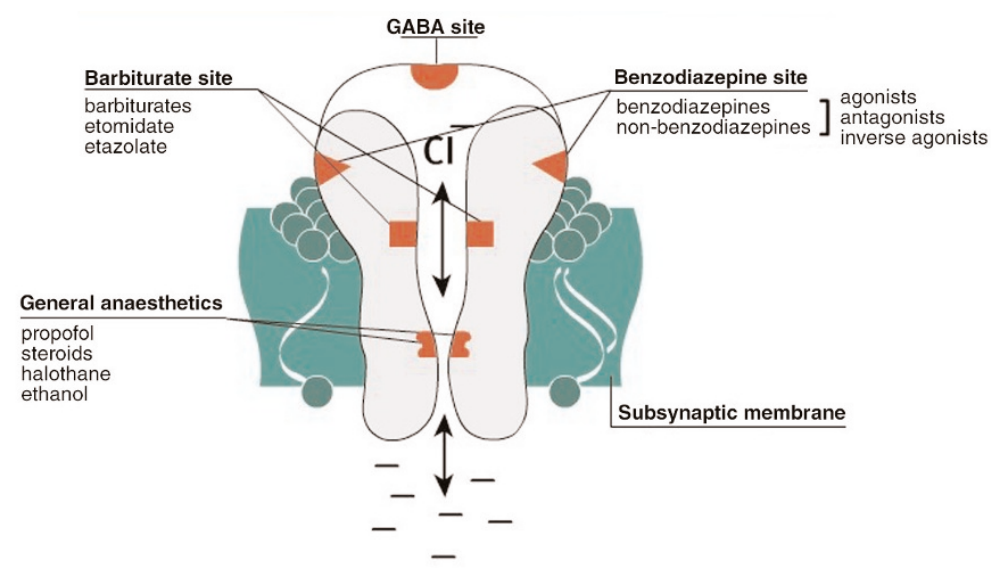

Functional binding sites on the GABA receptor. Adapted from the PACT Sedation module (European Society of Intensive Care Medicine www.esicm.org). Reprinted from Richards G, Schoch P, Haefely W: Benzodiazepine receptors: new vistas. Seminars in Neuroscience 1991, 3:191-203. (C) 1991 with permission from Elsevier.

whenever a patient exhibits an unexplained anion gap, unexplained metabolic acidosis, hyperosmolality, and/or clinical deterioration [21]. In addition, lorazepam has been found to be an independent risk factor for transition of ICU patients into delirium [22].

Barr and colleagues [23] investigated the pharmacokinetics and pharmacodynamics of midazolam and lorazepam administered as continuous intravenous infusions for postoperative sedation in surgical ICU patients. During the maintenance period of sedation, patients in the lorazepam group were optimally sedated (Ramsay Sedation Scale score 3 or 4) only $49 \%$ of the time, as compared with $69 \%$ of the time for midazolam. Patients receiving lorazepam were also more often deeply sedated (Ramsay Sedation Scale score 5 or 6) than those receiving midazolam. Patents who received lorazepam emerged from sedation more slowly and underwent extubation much later than did patients receiving midazolam ( 8.7 hours versus 3 hours and 21.2 hours versus 5.4 hours, respectively). It was also shown that older patients required much lower plasma concentrations of benzodiazepine to achieve levels of sedation comparable to those in younger patients.

\section{Propofol}

Propofol is a unique sedative-hypnotic agent with a rapid onset and offset of action. Like the benzodiazepines, propofol acts on the GABA receptor, although the site of action on this receptor is different (Fig. 1). Propofol is hydrophobic and so it is formulated in an oil-in-water emulsion. Pain at the injection site occurs in up to two-thirds of patients when propofol is injected into small veins.

The lipophilic properties of propofol allow it to cross the bloodbrain barrier rapidly. Metabolism of propofol occurs primarily by conjugation in the liver to inactive metabolites, which are eliminated by the kidneys. Hepatic and renal diseases have little impact on the pharmacokinetics of propofol. Hypotension attributed to systemic vasodilation is a well known adverse effect of propofol, especially in hypovolemic patients. Hypertriglyceridemia in ICU patients receiving propofol infusions occurs rarely and is typically associated with high propofol infusion rates, concurrent administration of parenteral lipids for nutrition, or baseline hypertriglyceridemia. Propofol infusion syndrome is a rare but serious and potentially fatal adverse effect, typically seen with infusion rates $>5 \mathrm{mg} / \mathrm{kg} / \mathrm{h}$ for more than 48 hours [24]. This syndrome is characterized by dysarhythmias, heart failure, metabolic acidosis, hyperkalemia and rhabdomyolysis, and it carries a mortality rate of up to $85 \%$. The pathophysiology of this syndrome is currently unclear, but it has been speculated that a combination of high-dose propofol with endogenous as well as exogenously administered catecholamines and/or glucocorticoids may act as a trigger. Treatment consists of immediate cessation of propofol administration, and correction of hemodynamic and metabolic abnormalities. In order to minimize the risk for this syndrome, propofol dosage should be under $4 \mathrm{mg} / \mathrm{kg}$ per hour for a maximum of 7 days [24]. Like benzodiazepines, propofol also appears to be an effective anticonvulsant.

The pharmacokinetics of propofol in critically ill patients depend on various factors. Barr and colleagues [25] studied the pharmacology of propofol infusions in ICU patients and demonstrated that the offset of sedation varies considerably, being a function of depth of sedation, duration of the infusion, and patient size and body composition. By integrating the results of the studied patients, they developed a dosing regimen for propofol sedation in ICU patients. For a typical ICU patient, the emergence times from light sedation (to decrease the Ramsey Sedation Scale score from 3 to 2) remained fairly rapid (<35 minutes) for propofol infusions 
lasting 3 days or less. For infusions lasting up to 14 days, the emergence time was 3.5 hours. In contrast, the emergence times from deep sedation (to decrease the Ramsey Sedation Scale score from 5 to 2) was 25 hours for a 24-hour infusion, and increased to about 3 days for propofol infusions lasting 7 to 14 days.

In a meta-analysis, Ostermann and colleagues [26] included 32 studies of sedation in the ICU (20 studies on short-term and 14 studies on long-term sedation). They found that propofol was at least as effective for sedation as was midazolam, and resulted in a shorter interval to extubation, but it was associated with increased risk for hypotension and higher cost. In contrast, Kress and coworkers [1] found no difference in the duration of mechanical ventilation and length of stay in the ICU or hospital between midazolam and propofol in mechanically ventilated ICU patients. One year later, the results of a multicenter trial conducted in four Canadian ICUs were published [27]; in this trial sedation with propofol was compared with midazolam sedation in critically ill patients. They found that propofol sedation allowed for more rapid tracheal extubation than when midazolam sedation was used but this did not result in earlier ICU discharge. Comparative studies evaluating the ability of propofol sedation to accelerate ICU discharge have yielded contradictory results. The reason for this is not yet known and randomized controlled trials are warranted.

\section{Dexmedetomidine}

Dexmedetomidine is a centrally acting $\alpha_{2}$-agonist with sedative and analgesic properties; it is similar to clonidine but has much greater $\alpha_{2}$ to $\alpha_{1}$ binding affinity. The sedative properties are facilitated through the locus coeruleus site in the CNS, and the analgesic effects may occur via activation of the $\alpha_{2}$ receptors by accentuating the action of opioids [28]. This class of drugs causes no significant effect on respiratory drive, even when used with opioids. Dexmedetomidine has a biphasic effect on the cardiovascular system. The initial bolus injection is associated with vasoconstrictive effects, causing bradycardia and hypertension. Continuous infusion is associated with hypotension secondary to vasodilation caused by central sympatholysis.

Most studies involving dexmedetomidine have evaluated postoperative ICU patients and demonstrated efficacy for short-term sedation and analgesic sparing [29]. Although dexmedetomidine is labeled in some countries only for sedation of less than 24 hours, the drug has not been extensively studied as an agent for long-term administration to critically ill, mechanically ventilated patients [30].

\section{Volatile sedation}

Isoflurane has successfully been used for sedation in ventilatordependent ICU patients. Several studies compared isoflurane with midazolam or with propofol [31-33] and found adequate sedation with predictable and quick awakening, without reported tolerance or withdrawal symptoms. Initially, the lack of necessary equipment led to limited use of volatile anesthetics as sedative agents in the ICU, but technologic advances, especially the introduction of the AnaConDa ${ }^{\circledR}$ filter (Hudson RCl, Uppsland Väsby, Sweden), have greatly simplified administration of volatile anesthetic agents in this setting. This device is connected between the patient and a normal ICU ventilator, and it maintains $90 \%$ of the volatile anesthetic inside the patient, analogous to the action of a heat-moisture exchanger [34]. It has been shown that isoflurane inhalation via this device was a safe and effective method of sedation in ICU patients, with significantly shorter awakening times (<25 minutes) compared with midazolam (range 57 to 837 minutes) [35]. Although volatile anesthetics are not licensed for use for sedation in the ICU, volatile sedation in the ICU appears a promising alternative to intravenous sedatives for mechanically ventilated adult patients in the ICU, but more clinical studies are warranted.

\section{Conclusion}

It appears that optimizing analgesia and sedation protocols is more important than seeking the ideal agent. The ideal regimen would provide adequate pain control and be easy to titrate, with a rapid onset and offset of action and without accumulation; it should also be cost-effective by improving quality of care, reducing the time spent on mechanical ventilation, or reducing the length of stay in the ICU. At present, none of the agents currently in use fulfills all of these requirements. In changing from deep to light sedation and from hypnotic-based to analgesic-based sedation, a short-acting opioid may be of importance. In addition, a relatively new agent for ICU sedation, the $\alpha_{2}$-agonist dexmedetomidine, is promising because of its combination of sedative and analgesic properties but with less effect on respiratory drive. However, we currently lack efficacy and safety data for longer dexmedetomidine infusions. Inhalational sedation may also be advantageous in ICU sedation. For example, isoflurane is among the safest anesthetic drugs, with almost complete elimination via exhalation after administration is discontinued [36]. It is therefore independent of renal or liver function, which may be of particular importance in ICU sedation. Despite new devices for use of volatile anesthetics in the ICU, more studies are needed to confirm their efficacy and safety. Thus, clinicians should continue to search for the optimal regimen, using both new and old agents in various combinations.

\section{Competing interests}

DG declares no competing interests. JB has received grants and reimbursements from GlaxoSmithKline but has not received any financial support related to this article.

\section{Acknowledgements}

This article is part of Critical Care Volume 12 Supplement 3: Analgesia and sedation in the ICU. The full contents of the supplement are available online at http://ccforum.com/supplements/12/S3. 
Publication of the supplement has been funded by an unrestricted grant from GlaxoSmithKline.

\section{References}

1. Kress JP, Pohlman AS, O'Connor MF, Hall JB: Daily interruption of sedative infusions in critically ill patients undergoing mechanical ventilation. $N$ Engl J Med 2000, 342:1471-1477.

2. Karabinis A, Mandragos K, Stergiopoulos S, Komnos A, Soukup J, Speelberg B, Kirkham AJ: Safety and efficacy of analgesiabased sedation with remifentanil versus standard hypnoticbased regimens in intensive care unit patients with brain injuries: a randomised, controlled trial. Crit Care 2004, 8: R268-R280.

3. Park G, Lane M, Rogers S, Bassett P: A comparison of hypnotic and analgesic based sedation in a general intensive care unit. BrJ Anaesth 2007, 98:76-82.

4. Guay J: The benefits of adding epidural analgesia to general anesthesia: a metaanalysis. J Anesth 2006, 20:335-340.

5. Liu SS, Block BM, Wu CL: Effects of perioperative central neuraxial analgesia on outcome after coronary artery bypass surgery: a meta-analysis. Anesthesiology 2004, 101:153-161.

6. Bulger EM, Edwards T, Klotz P, Jurkovich GJ: Epidural analgesia improves outcome after multiple rib fractures. Surgery 2004, 136:426-430.

7. Bernhardt A, Kortgen A, Niesel HCh, Goertz A: Using epidural anestheia in patients with acute pancreatitis: prospective study of 121 patients. Anaesthesiol Reanim 2002, 27:16-22.

8. Low JHS: Survey of epidural analgesia management in general intensive care units in England. Acta Anaesthesiol Scand 2002, 46:799-805.

9. Soliman HM, Melot C, Vincent JL: Sedative and analgesic practice in the intensive care unit: the results of a European survey. Br J Anaesth 2001, 87:186-192.

10. Pasternak GW, Bodnar RJ, Clark JA, Inturrisi CE: Morphine-6glucuronide, a potent mu agonist. Life Sci 1987, 41:28452849.

11. Frances B, Gout R, Campistron G, Panconi E, Cros J: Morphine6-glucuronide is more mu-selective and potent in analgesic tests than morphine. Prog Clin Biol Res 1990, 328:477-480

12. Jacobi J, Fraser GL, Coursin DB, Riker RR, Fontaine D, Wittbrodt ET, Chalfin DB, Masica MF, Bjerke HS, Coplin WM, Crippen DW, Fuchs BD, Kelleher RM, Marik PE, Nasraway SA Jr, Murray MJ, Peruzzi WT, Lumb PD; Task Force of the American College of Critical Care Medicine (ACCM) of the Society of Critical Care Medicine (SCCM), American Society of Health-System Pharmacists (ASHP), American College of Chest Physicians: Clinical practice guidelines for the sustained use of sedatives and analgesics in the critically ill adult. Crit Care Med 2002, 30: 119-141.

13. Beers R, Camporesi E: Remifentanil update: clinical science and utility. CNS Drugs 2004, 18:1085-1104.

14. Muellejans B, Lopez A, Cross MH, Bonome C, Morrison L, Kirkham AJ: Remifentanil versus fentanyl for analgesia based sedation to provide patient comfort in the intensive care unit: a randomized, double-blind controlled trial. Crit Care 2004, 8: R1-R11.

15. Dahaba AA, Grabner T, Rehak PH, List WF, Metzler H: Remifentanil versus morphine analgesia and sedation for mechanically ventilated critically ill patients: a randomized double blind study. Anesthesiology 2004, 101:640-646.

16. Breen D, Karabinis A, Malbrain M, Morais R, Albrecht S, Jarnvig IL, Parkinson P, Kirkham AJT: Decreased duration of mechanical ventilation when comparing analgesia-based sedation using remifentanil with standard hypnotic-based sedation for up to 10 days in intensive care unit patients: a randomised trial. Crit Care 2005, 9:R200.

17. Bakker J, Mulder P: Remifentanil shortens duration of mechanical ventilation and ICU stay. Intensive Care Med 2006, 32 (Suppl):S85

18. Pitsiu M, Wilmer A, Bodenham A, Breen D, Bach V, Bonde J, Kessler P, Albrecht S, Fisher G, Kirkham A: Pharmacokinetics of remifentanil and its major metabolite, remifentanil acid, in ICU patients with renal impairment. BrJ Anaesth 2004, 92:493-503.

19. Breen D, Wilmer A, Bodenham A, Bach V, Bonde J, Kessler P Albrecht S, Shaikh S: Offset of pharmacodynamic effects and safety of remifentanil in intensive care unit patients with various degrees of renal impairment. Crit Care 2004, 8:R21R30.

20. Amrein R, Hetzel W, Hartmann D, Lorscheid T: Clinical pharmacology of flumazenil. Eur J Anaesthesiol Supp/ 1988, 2:65-80.

21. Wilson KC, Reardon C, Theodore AC, Farber HW: Propylene glycol toxicity: a severe iatrogenic illness in ICU patients receiving iv benzodiazepines. Chest 2005, 128:1674-1681.

22. Pandharipande P, Shintani A, Peterson J, Pun BT, Wilkinson GR, Dittus RS, Bernard GR, Ely EW: Lorazepam is an independent risk factor for transitioning to delirium in intensive care unit patients. Anesthesiology 2006, 104:21-26.

23. Barr J, Zomorodi K, Bertaccini EJ, Shafer SL, Geller E: A doubleblind, randomized comparison of i.v. lorazepam versus midazolam for sedation of ICU patients via a pharmacologic model. Anesthesiology 2001, 95:286-298.

24. Wappler F: The propofol infusion syndrome [in German]. Deutsches Arzteblatt 2006, 11:A705-A710.

25. Barr J, Egan TD, Sandoval NF, Zomorodi K, Cohane C, Gambus PL, Shafer SL: Propofol dosing regimens for ICU sedation based upon an integrated pharmacokinetic-pharmacodynamic model. Anesthesiology 2001, 95:324-333.

26. Ostermann ME, Keenan SP, Seiferling RA, Sibbald WJ: Sedation in the intensive care unit: a systematic review. JAMA 2000, 283:1451-1459.

27. Hall RI, Sandham D, Cardinal P, Tweeddale M, Moher D, Wang X, Anis AH; Study Investigators: Propofol vs midazolam for ICU sedation: a Canadian multicenter randomized trial. Chest 2001, 119:1151-1159.

28. Szumita PM, Baroletti SA, Anger KE, Wechsler ME: Sedation and analgesia in the intensive care unit: evaluating the role of dexmedetomidine. Am J Health Syst Pharm 2007, 64:37-44.

29. Martin E, Ramsay G, Mantz J, Sum-Ping STJ: The role of the $\alpha_{2}$ adrenoceptor agonist dexmedetomidine in postsurgical sedation in the intensive care. J Intensive Care Med 2003, 18: 29-41.

30. Venn RM, Newman PJ, Grounds RM: A phase II study to evaluate the efficacy of dexmedetomidine for sedation in the intensive care unit. Intensive Care Med 2003, 29:201-207.

31. Kong KL, Willarts SM, Prys-Roberts C: Isoflurane compared with midazolam for sedation in the intensive care unit. $B M J$ 1989, 298:1277-1280.

32. Spencer EM, Willatts SM: Isoflurane for prolonged sedation in the intensive care unit: efficacy and safety. Intensive Care Med 1992, 18:415-421.

33. Millane TA, Bennet ED, Grounds RM: Isoflurane and propofol for long-term sedation in the intensive care unit: a crossover study. Anaesthesia 1992, 47:768-774.

34. Berton J, Sargentini C, Nguygen JL, Belii A, Beydon L. AnaConDa reflection filter: bench and patient evaluation of safety and volatile anesthetic conservation. Anesth Analg 2007, 104:130134.

35. Sackey PV, Martling C-R, Granath F, Radell PJ: Prolonged isoflurane sedation of intensive care unit patients with the anesthetic conserving device. Crit Care Med 2004, 32:22412246.

36. Carpenter RL, Eger El 2nd, Johnson BH, Unackat JP, Sheiner LB: The extent of metabolism of inhaled anesthetics in humans. Anesthesiology 1986; 65:201-205.

Disclaime

This article is part of Critical Care Volume 12 Supplement 3: Analgesia and sedation in the ICU. Publication of the supplement has been funded by an unrestricted grant from GlaxoSmithKline. GlaxoSmithKline has had no editorial control in respect of the articles contained in this publication.

The opinions and views expressed in this publication are those of the authors and do not The opinions and views expressed in this publication are those of the authors and do not
constitute the opinions or recommendations of the publisher or GlaxoSmithKline. Dosages, constitute the opinions or recommendations of the publisher or GlaxoSmithKline. Dosages,
indications and methods of use for medicinal products referred to in this publication by the authors may reflect their research or clinical experience, or may be derived from professional literature or other sources. Such dosages, indications and methods of use may not reflect the prescribing information for such medicinal products and are not recommended by the publisher or GlaxoSmithKline. Prescribers should consult the prescribing information approved for use in their country before the prescription of any medicinal product. Whilst every effort is made by the publisher and editorial board to see that no inaccurate or misleading data, opinion, or statement appear in this publication, they wish to make it clear that the data and opinions appearing in the articles herein are the sole responsibility of the contributor concerned.

Accordingly, the publishers, the editor and editorial board, GlaxoSmithKline, and their respective employees, officers and agents accept no liability whatsoever for the consequences of such inaccurate or misleading data, opinion or statement. 